\title{
Cortical drive to breathe in amyotrophic lateral sclerosis: a dyspnoea-worsening defence?
}

\author{
Marjolaine Georges ${ }^{1,2,3}$, Elise Morawiec ${ }^{1,2}$, Mathieu Raux ${ }^{1,2,4}$, \\ Jésus Gonzalez-Bermejo ${ }^{1,2,3}$, Pierre-François Pradat ${ }^{5}$, Thomas Similowski ${ }^{1,2,3,6}$ \\ and Capucine Morélot-Panzini ${ }^{1,2,3,6}$
}

\begin{abstract}
Affiliations: 'UMRS1158 Neurophysiologie Respiratoire Expérimentale et Clinique, Sorbonne Universités, UPMC Université Paris 06, INSERM, Paris, France. ${ }^{2}$ Service de Pneumologie et Réanimation Médicale (Département R3S), AP-HP, Groupe Hospitalier Pitié-Salpêtrière Charles Foix, Paris, France. ${ }^{3}$ Service de Pneumologie et Réanimation Respiratoire, Centre Hospitalier Universitaire de Dijon, Dijon, France. ${ }^{4}$ Département d'Anesthésie et Réanimation, AP-HP, Groupe Hospitalier Pitié-Salpêtrière Charles Foix, Paris, France. ${ }^{5}$ Service de Neurologie, AP-HP, Groupe Hospitalier Pitié-Salpêtrière Charles Foix, Paris, France. ${ }^{6}$ Both authors contributed equally.
\end{abstract}

Correspondence: Capucine Morélot-Panzini, Service de Pneumologie et Réanimation Médicale (Département R3S), Groupe Hospitalier Pitié-Salpêtrière Charles Foix, 47-83 Bd de l'Hôpital, 75651 Paris Cedex 13, France. E-mail: capucine.morelotapsl.aphp.fr

ABSTRACT Amyotrophic lateral sclerosis (ALS) is a neurodegenerative disease causing diaphragm weakness that can be partially compensated by inspiratory neck muscle recruitment. This disappears during sleep, which is compatible with a cortical contribution to the drive to breathe. We hypothesised that ALS patients with respiratory failure exhibit respiratory-related cortical activity, relieved by noninvasive ventilation (NIV) and related to dyspnoea.

We studied 14 ALS patients with respiratory failure. Electroencephalographic recordings (EEGs) and electromyographic recordings of inspiratory neck muscles were performed during spontaneous breathing and NIV. Dyspnoea was evaluated using the Multidimensional Dyspnea Profile.

Eight patients exhibited slow EEG negativities preceding inspiration (pre-inspiratory potentials) during spontaneous breathing. Pre-inspiratory potentials were attenuated during NIV ( $\mathrm{p}=0.04)$. Patients without pre-inspiratory potentials presented more advanced forms of ALS and more severe respiratory impairment, but less severe dyspnoea. Patients with pre-inspiratory potentials had stronger inspiratory neck muscle activation and more severe dyspnoea during spontaneous breathing.

ALS-related diaphragm weakness can engage cortical resources to augment the neural drive to breathe. This might reflect a compensatory mechanism, with the intensity of dyspnoea a negative consequence. Disease progression and the corresponding neural loss could abolish this phenomenon. A putative cognitive cost should be investigated.

@ERSpublications

Awake ALS patients with respiratory failure mobilise cortical resources to keep breathing; dyspnoea is the ransom http://ow.ly/XGhaf

This article has been revised according to the correction published in the August 2016 issue of the European Respiratory Journal.

Editorial comment in Eur Respir J 2016; 47: 1632-1634.

Received: Oct 112015 | Accepted after revision: Jan 192016 | First published online: April 132016

Support statement: The study was supported by public funding, including a "Legs Poix" grant from the Chancellerie de l'Université de Paris, grant ANR-11-EMMA-030-01 from Agence Nationale de la Recherche and the French government's Investissement d'Avenir ANR-10-AIHU 06 programme. Funding information for this article has been deposited with FundRef.

Conflict of interest: None declared.

Copyright OERS 2016 


\section{Introduction}

Amyotrophic lateral sclerosis (ALS) is a neurodegenerative disease characterised by progressive loss of upper and lower motor neurons, resulting in wasting of skeletal muscles, including the respiratory muscles. Respiratory muscle weakness impairs quality of life in relation to sleep-related respiratory disorders and dyspnoea [1], and respiratory failure is a prominent cause of ALS-related death $[2,3]$.

The ALS-related degenerative process involves all respiratory muscle groups, with ensuing inspiratory and expiratory muscle weakness. This reduces ventilatory capacity and deteriorates respiratory mechanics, mostly through the impairment of cough, which leads to airway mucus encumbrance and atelectasis. As a result, a load-capacity imbalance develops, with signs of diaphragmatic dysfunction a prominent feature of the disease. ALS patients with diaphragm weakness often exhibit intense inspiratory neck muscle recruitment during wakefulness $[4,5]$, which is interpreted as compensatory in nature. This activity decreases or disappears during sleep, leading to hypoventilation [6]. As in the case of other wakefulness-dependent respiratory phenomena, it can be hypothesised that inspiratory muscle recruitment during wakefulness proceeds from a cortical contribution to the neural drive to breathe. A similar hypothesis has been proposed to explain the maintenance of ventilation during wakefulness in patients with defective automatic breathing, such as patients suffering from congenital central alveolar hypoventilation due to a PHOX2B mutation [7]. In such patients, electroencephalographic recordings (EEGs) time-locked to the spontaneous ventilatory activity have evidenced specific pre-inspiratory potentials resembling those that are observed during the preparation and execution of voluntary movements [7]. In healthy people, there is normally no respiratory-related cortical activity during quiet breathing, which means that pre-inspiratory potentials cannot be detected in this condition. They appear in the presence of a mechanical inspiratory load $[8,9]$, and reflect the activation and subsequent automatisation of respiratory cortico-subcortical networks involving the supplementary motor area (SMA) [10]. In the context of intrinsic inspiratory loads, they have been observed during normal breathing in patients suffering from severe forms of the obstructive sleep apnoea syndrome [11], and have been interpreted as contributing to the absence of upper airway obstructive events during wakefulness.

We therefore hypothesised that some ALS patients with respiratory failure would exhibit a respiratory-related cortical activity in the form of pre-inspiratory potentials, an activity that would be abolished or attenuated by noninvasive ventilation (NIV). We anticipated heterogeneous findings because of ALS-related cortical degeneration as a possible confounding factor. Because the presence of pre-inspiratory potentials in reaction to respiratory loading bears a relationship with respiratory discomfort $[8,12]$, we also predicted that a putative respiratory-related cortical activity would influence dyspnoea.

\section{Material and methods Patients}

This study was conducted in the home mechanical ventilation unit of the respiratory medicine department at a 1600-bed tertiary university hospital. This unit is part of the local reference ALS multidisciplinary centre. The study was conducted in accordance with the guidelines of the Declaration of Helsinki and was part of an experimental programme approved by the appropriate French regulatory and ethical authority (Comité de Protection des Personnes). Patients received detailed information about the methods used and provided written consent to participate. The exact purpose of the study was explained after completion of the measurement to ensure that participants were as naïve as possible and to avoid bias. The inclusion criteria were as follows: 1) a probable or definite diagnosis of ALS according to the revised El Escorial criteria [13]; 2) overt respiratory failure with clear recruitment of inspiratory neck muscles during quiet breathing, at least when supine; and 3) previously established NIV. The exclusion criteria were as follows: 1) coexistence of any other respiratory diagnosis; 2) cumulative tobacco consumption >10 pack-years; 3) history of acute respiratory symptoms during the past 6 weeks; 4) gross cognitive impairment; and 5) end-stage disease.

Given the exploratory nature of the study, no statistical power calculation was performed, and a convenience sample of 14 patients was studied (table 1). Two patients had an initial bulbar form of ALS and one had an initial respiratory form. All patients received $50 \mathrm{mg}$ of riluzole twice daily. None were gastrostomised. NIV settings were as follows: positive inspiratory pressure $17 \mathrm{cmH}_{2} \mathrm{O}$ [14-20], positive expiratory pressure $8 \mathrm{cmH}_{2} \mathrm{O}$ [4-10] and back-up respiratory rate $14 \mathrm{bpm}$ [12-16].

\section{Experimental protocol}

Patients were studied in a sitting position that provided full support to the back, arms, neck and head. During the entire experiment, they watched an emotionally neutral movie (e.g. animal documentary) or television show to distract their attention from the experimental set-up and from their breathing. They were instructed to relax but to keep their eyes open to avoid any risk of falling asleep and to minimise eye movements. 
TABLE 1 Patient characteristics at the time of the study

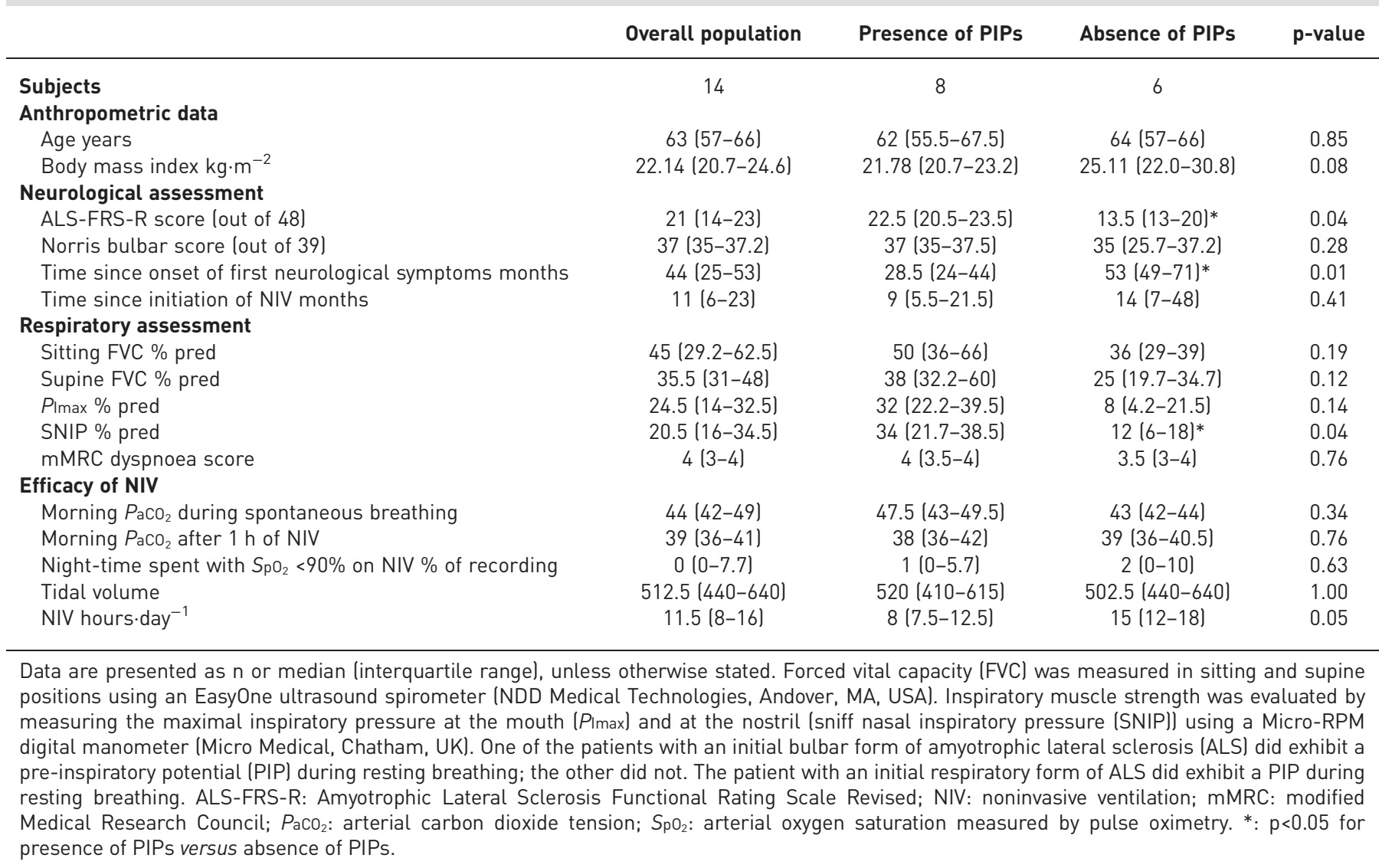

Ventilatory variables, electromyograms (EMGs) and EEGs were first recorded during spontaneous room air breathing and then on NIV (Stellar, spontaneous-timed pressure support mode; ResMed, Bella Vista, Australia). In each of these conditions, the first $10 \mathrm{~min}$ were discarded to allow for stabilisation, and the following $20 \mathrm{~min}$ were kept for analysis. There was $\sim 5-10 \mathrm{~min}$ between the two recordings.

\section{Measurements}

Ventilatory variables

During spontaneous breathing, ventilatory airflow was recorded through a nasal cannula connected to a $\pm 2 \mathrm{cmH}_{2} \mathrm{O}$ linear differential pressure transducer (DP-45-18; Validyne, Northridge, CA, USA). This approach was chosen to minimise both apparatus-related inspiratory loading and apparatus-related focusing on breathing, both factors being liable to give rise to artefactual respiratory-related cortical activity (see discussion in [12]).

During NIV, the subjects breathed through the same facemask as they used at home (in all cases a QuattroFX; ResMed) connected to their ventilator and attached in series to a low-resistance pneumotachograph connected to a $\pm 2 \mathrm{cmH}_{2} \mathrm{O}$ linear differential pressure transducer (DP-45-18) to measure ventilatory airflow. Airway pressure was measured using a $\pm 140 \mathrm{cmH}_{2} \mathrm{O}$ differential pressure transducer (DP 15-32; Validyne).

\section{Dyspnoea}

Dyspnoea was evaluated using the French version of the Multidimensional Dyspnea Profile (MDP) after each recording session (spontaneous breathing and NIV), the patients being asked to focus on the past $30 \mathrm{~s}$ in each case [14]. This questionnaire comprises 11 items: one item (A1) assesses the unpleasantness of dyspnoea on a $0-10$ visual analogue scale (from "neutral" to "unbearable"); five items assess the sensory dimension of dyspnoea in terms of quality and intensity (0-10); and five items assess the affective dimension of dyspnea in terms of quality and intensity $(0-10)$. We calculated an "immediate perception domain" score $(\mathrm{S})$ as the sum of the A1 intensity and the intensities of the five sensory descriptors and an "emotional response domain" score (A2) as the sum of the five emotional descriptors $[14,15]$. 
Electroencephalographic activity

EEG was recorded using active surface electrodes placed at scalp positions Fp1, Fpz, Fp2, F3, Fz, F4, Fcz, $\mathrm{C} 3, \mathrm{Cz}, \mathrm{C} 4, \mathrm{P} 3$ and $\mathrm{P} 4$ on the basis of the international 10-20 electrode placement system using a 12-electrode cap installed after application of a conductive gel (EasyCap; Brain Products, Gilching, Germany). Electrode impedances were monitored and maintained at $<5 \mathrm{k} \Omega$. The EEG signal was digitised at $2000 \mathrm{~Hz}$, recorded using V-Amp software (Brain Products) and filtered $(0.01-5 \mathrm{~Hz})$. EEG data were stored for offline analysis.

Surface electromyographic recordings of inspiratory neck muscles

Surface recordings of the EMG activity of inspiratory neck muscles were obtained using a pair of silver cup electrodes placed over the anatomical landmark of the middle scalene, $2 \mathrm{~cm}$ above the clavicle. The EMG signal was fed to an amplifier (Neuropack electromyograph; Nihon Kohden, Tokyo, Japan) with a $2 \mathrm{kHz}$ sampling rate and was filtered between 20 and $3000 \mathrm{~Hz}$. EEGs were digitised at $2 \mathrm{~Hz}$ and stored for offline analysis (LabChart version 7.0; AD Instruments, Oxford, UK).

\section{Signal processing}

Electroencephalographic data

Offline, EEG signals were referenced to the average of the electrodes. The EEG was split into $3 \mathrm{~s}$ epochs extending from $2.5 \mathrm{~s}$ before to $0.5 \mathrm{~s}$ after the onset of inspiration, as determined from the flow trace. Based on previous experiments [16], we have determined that $\sim 80$ epochs must be averaged to clearly identify pre-inspiratory potentials, which requires the recording of $\sim 120$ epochs to account for rejection criteria. The EEG recording therefore lasted $\geqslant 120$ breaths at the end of the steady-state period. All epochs exhibiting EEG artefacts, spurious EEG activity exceeding $20 \%$ of the baseline background signal or intense electro-oculographic activity were discarded (median rejection rate 38\% [21-39]). On the averaged tracings, a slow negative shift starting between 2 and $0.5 \mathrm{~s}$ before inspiration was identified as pre-motor activity based on visual inspection by two observers blinded to the recording condition. Discrepancies were resolved by a third observer, also blinded to the recording condition. When a pre-inspiratory potential was considered to be present, its slope, amplitude and area under the curve (AUC) were determined. Finally, patients were grouped according to the presence or absence of pre-inspiratory potentials during spontaneous breathing, and a point-by-point ensemble averaging procedure was conducted among these groups.

\section{Electromyographic data}

The root mean square (RMS) of the inspiratory neck muscle EMG, reflecting the electrical energy spent by muscle contraction was calculated numerically using fixed 1-ms windows. For each condition in each subject, the beginning of inspiration was identified from the flow signal. The continuous EMG RMS signal was truncated into as many epochs as there were inspiratory efforts, with each period starting $1 \mathrm{~s}$ before the beginning of the corresponding inspiratory effort and ceasing $2 \mathrm{~s}$ after it ended; the epochs therefore contained the full inspiratory-related EMG activity [17, 18]. A set of 70-80 EMG epochs were then ensemble averaged, resulting in a mean EMG RMS envelope that was used for subsequent analysis [17, 18]. The averaged EMG RMS was used to measure the maximum EMG activity, the EMGAUC and the produce of EMGAUC $\times$ respiratory rate $(f \mathrm{R})$.

\section{Statistical analysis}

Statistical analysis was performed using SigmaStat software (Systat Software Inc., San Jose, CA, USA). Because data distributions were generally non-normal (Kolmogorov-Smirnov test), the data were summarised by the median and interquartile range, and nonparametric tests were used. The occurrence of pre-inspiratory potentials during spontaneous breathing and NIV were compared using Fisher's exact test. Spontaneous breathing and NIV were compared in terms of the presence of pre-inspiratory potentials using a Wilcoxon signed-rank test. Patients exhibiting pre-inspiratory potentials and those not exhibiting pre-inspiratory potentials were compared using a Mann-Whitney U-test. The relationship between dyspnoea ratings and inspiratory neck muscle EMG values was examined using Spearman's correlation coefficient. Comparisons were considered significant when $\mathrm{p}<0.05$.

\section{Results}

\section{Respiratory-related EEG activity}

Pre-inspiratory cortical potentials were present in the $\mathrm{Cz}$ derivation in eight patients during spontaneous resting breathing and absent in the remaining six patients (figs 1-3). Only two of these eight patients still exhibited pre-inspiratory potentials during NIV (OR 7.4, 95\% CI 1.0-92.8; $\mathrm{p}=0.046$ ) (figs 1 and 3 ). In these two cases, the persistent pre-inspiratory potentials had reduced amplitudes $(2.13 \mu \mathrm{V}$ during spontaneous breathing versus $1.43 \mu \mathrm{V}$ during NIV in one case; 5.51 versus $1.45 \mu \mathrm{V}$ in the other case). The area under the averaged EEG activity curve was also reduced (1601 versus $798 \mu \mathrm{V}^{2}$ and 3627 versus $865 \mu \mathrm{V}^{2}$, respectively), 


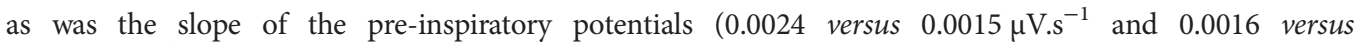
$0.0008 \mu \mathrm{V} . \mathrm{s}^{-1}$, respectively).

\section{Inspiratory neck muscle EMG activity}

Phasic inspiratory activity was clearly visible in almost all EMG recordings during spontaneous breathing (13 out of 14 patients; electrical interferences prevented analysis in one case) (figs 1 and 2), which is generally not observed in healthy people. Inspiratory neck muscle EMG activity was completely or almost completely abolished during NIV (fig. 1 and table 2) in all patients, including the two patients who exhibited a pre-inspiratory potential during spontaneous breathing and in whom NIV was not associated with complete disappearance of this potential.

\section{Dyspnoea}

Ratings of the MDP components are described in fig. 4. The patients reported intense dyspnoea during spontaneous breathing; "air hunger" and "work or effort" being the sensory descriptors most frequently used, and "anxiety" being the affective descriptor most frequently used. NIV almost completely relieved dyspnoea (fig. 4 and table 3). Of note, most patients considered it pointless to answer the "affective" questions after NIV, hence the absence of pre/post data for these items (fig. 4).

In the overall study population, a significant correlation was observed between the NIV-related decrease in the A1 score of the MDP and the corresponding decrease in the EMGAUC $\times f R$ product (fig. 5). No other correlation was observed between any of the dyspnoea scores (and their changes) and inspiratory neck muscle EMG activity (and its changes).

a)

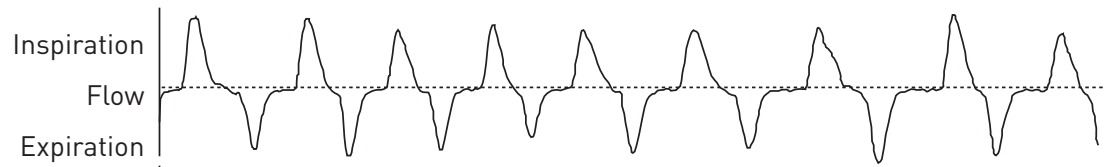

Inspiratory time-locked average $(0-2 \mathrm{~s})$
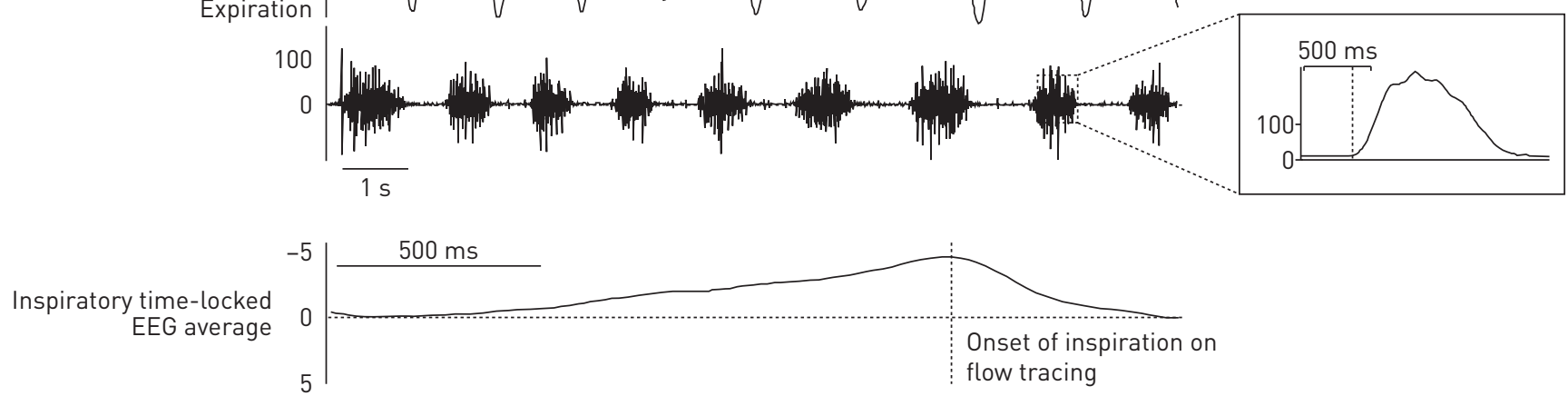

b)
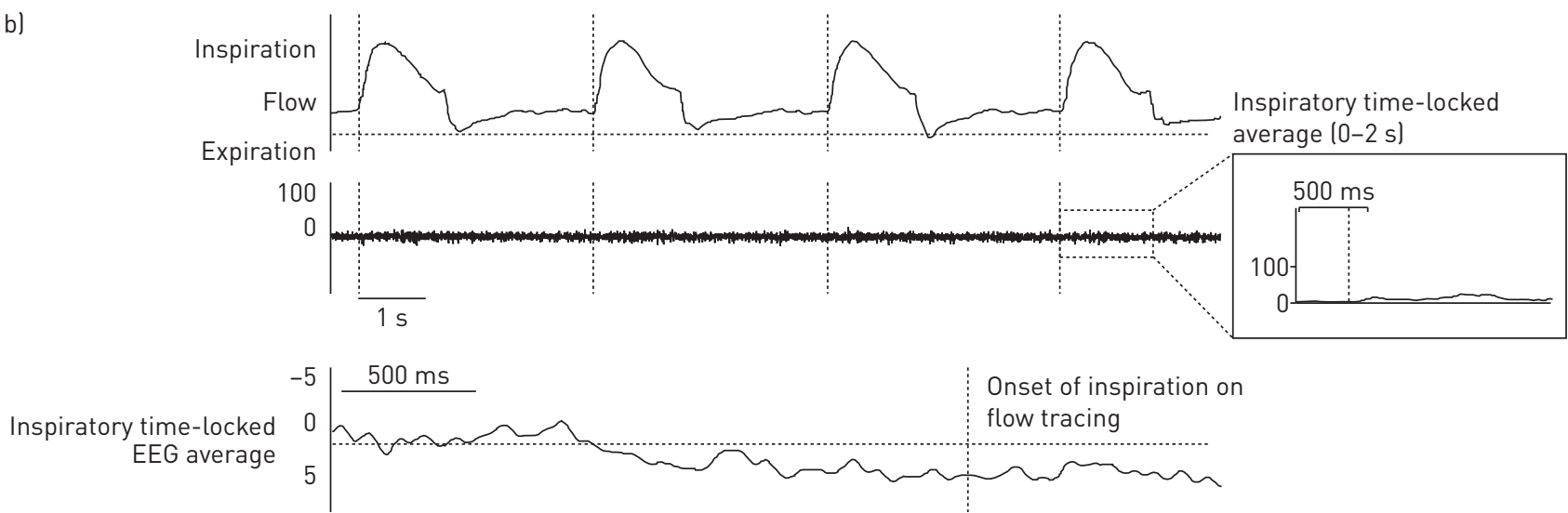

FIGURE 1 Inspiratory neck (scalene) muscle electromyogram (EMG) and electroencephalographic (EEG) detection of respiratory-related cortical activity in the form of pre-inspiratory potentials in one patient with amyotrophic lateral sclerosis during a) spontaneous breathing and b) noninvasive ventilation. Strong phasic inspiratory neck muscle EMG activity is clearly visible during spontaneous breathing, giving rise to a well-defined inspiratory envelope (right insert; the vertical dashed line indicates the onset of inspiration). This activity almost completely disappears on noninvasive ventilation. The inspiratory time-locked averaging of the EEG signal indicates a clearly visible pre-inspiratory potential during spontaneous breathing, while this type of potential is not observed during noninvasive ventilation. Note the dramatic decrease in respiratory frequency between spontaneous breathing and noninvasive ventilation. 
a)
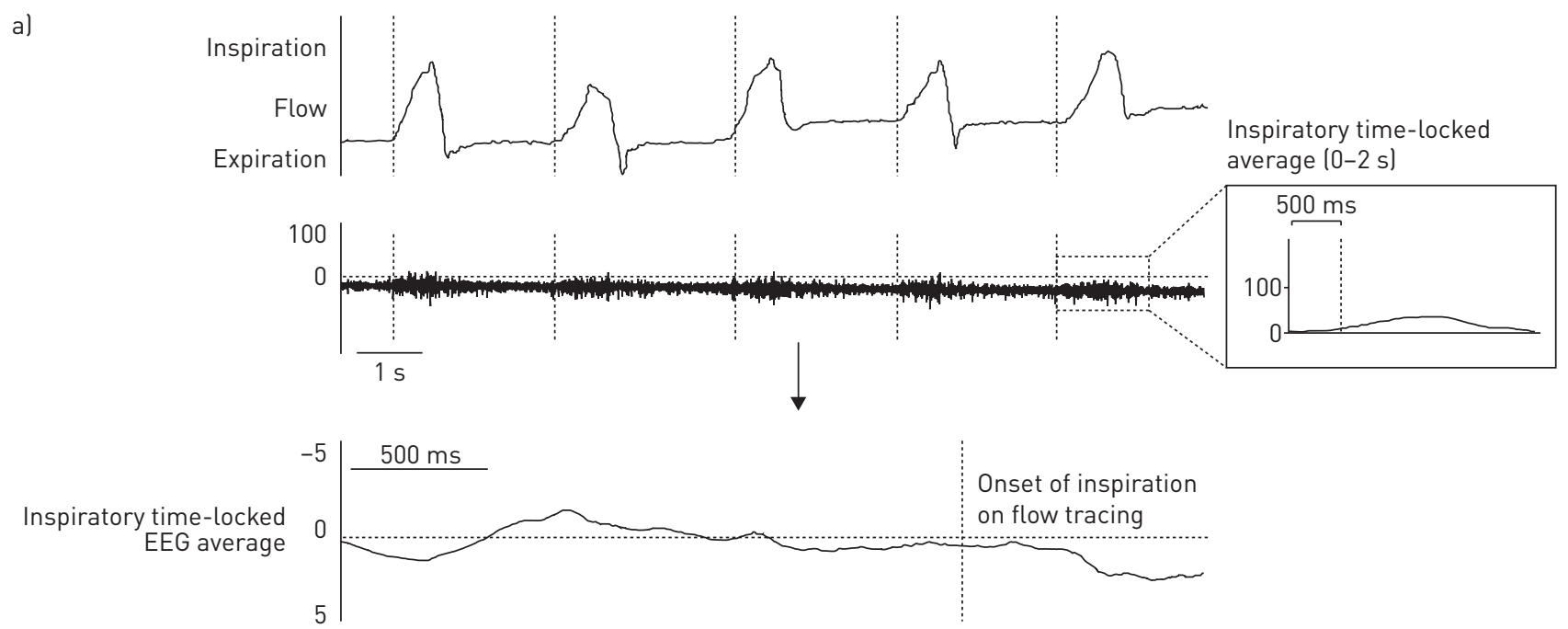

b)

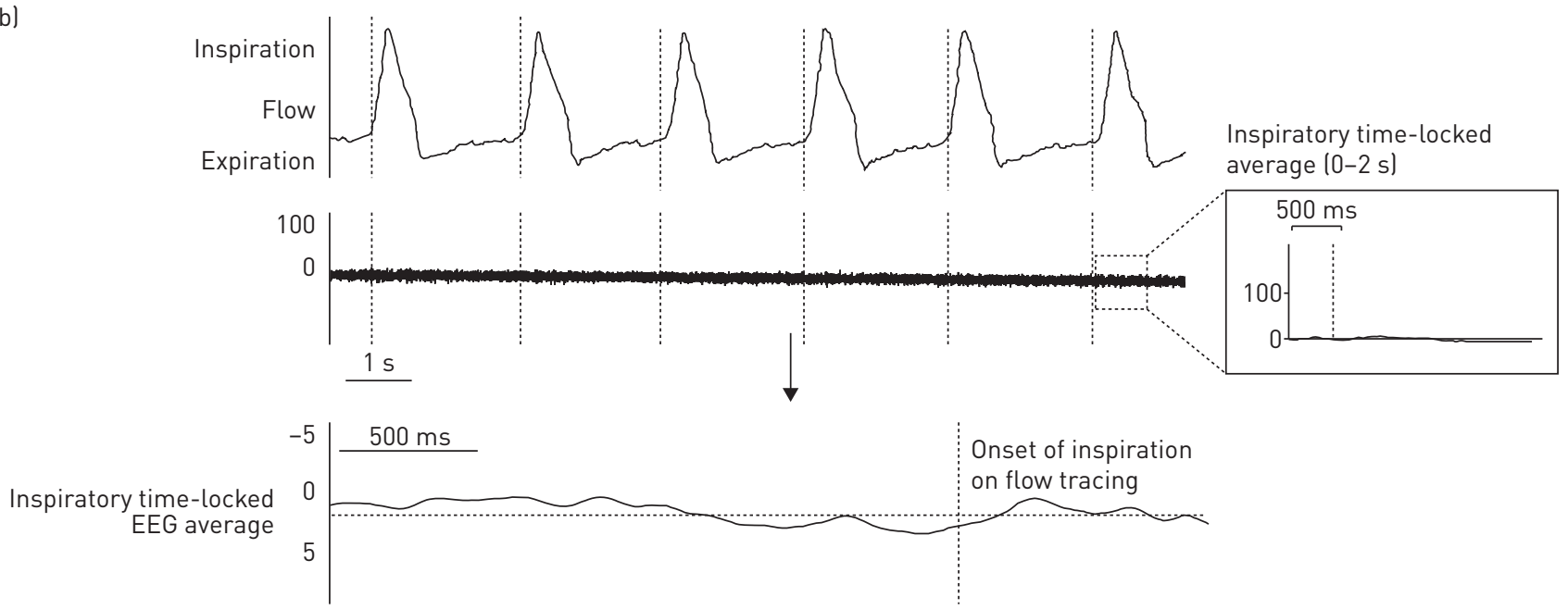

FIGURE 2 Inspiratory neck (scalene) muscle electromyogram (EMG) and electroencephalographic (EEG) detection of respiratory-related cortical activity in the form of pre-inspiratory potentials in one patient with amyotrophic lateral sclerosis not exhibiting this type of activity during a) spontaneous breathing and b) noninvasive ventilation. Phasic inspiratory neck muscle EMG activity is visible during spontaneous breathing, giving rise to a small inspiratory envelope (right insert; the vertical dashed line indicates the onset of inspiration). This activity almost completely disappears upon noninvasive ventilation. The inspiratory time-locked averaging of the EEG signal does not reveal any visible pre-inspiratory potential during either spontaneous breathing or noninvasive ventilation.

Comparison of patients with and without pre-inspiratory potentials during resting breathing Patients who did not exhibit pre-inspiratory potentials during spontaneous breathing had a more advanced form of ALS (table 1). They had significantly longer disease duration and significantly lower ALS Functional Rating Scale-Revised scores and sniff nasal inspiratory pressures; additionally, they used NIV for longer daily durations.

Patients who exhibited a pre-inspiratory potential during spontaneous breathing had higher EMGAUC $\times f \mathrm{R}$ values (a descriptor of the intensity of the drive to breathe) (table 2). These patients reported higher sensory dyspnoea ratings during spontaneous breathing, both for aggregated scores and "air hunger" and "work or effort" items considered separately. Emotional dyspnoea ratings were not significantly different between the two categories of patients (table 3).

\section{Discussion}

In line with our hypothesis, this study shows that ALS patients with respiratory failure can, in certain cases, exhibit respiratory-related cortical activity during resting breathing. This activity is associated with more intense dyspnoea, and disappears under NIV. 
a)

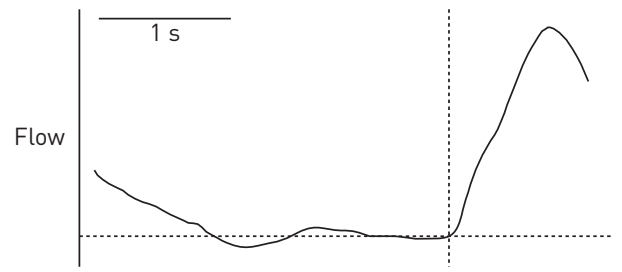

ii)

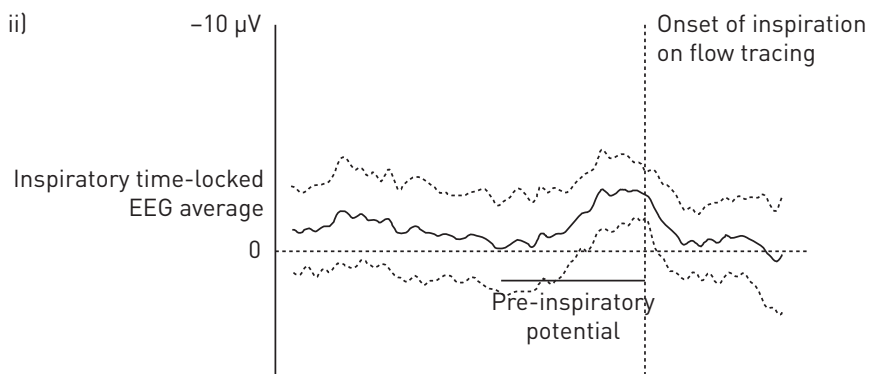

b)

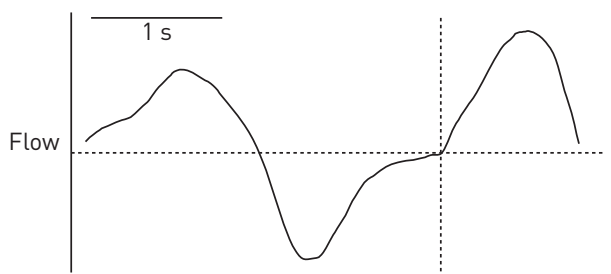

ii)

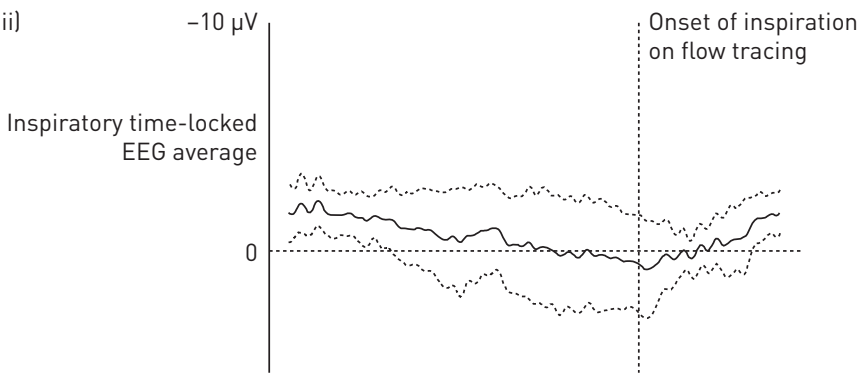

FIGURE 3 Ensemble averaging of the electroencephalographic (EEG) recordings obtained in a) the eight patients exhibiting pre-inspiratory potentials based on analysis of individual recordings: i) flow signal and ii) EEG signal; and b) the six patients not exhibiting pre-inspiratory potentials: i) flow signal and ii) EEG signal. EEG signal data are presented as mean \pm 1 SD.

\section{Analogy with inspiratory loading}

Resting ventilation is normally under the control of brainstem automatic neural activities [19]. Cortico-subcortical networks allow disruption of ventilatory automatisms during voluntary apnoea [20], voluntary breathing $[40,41]$ or speech [42]. Experimental studies have shown that some of these networks, which comprise the SMA $[10,43]$, become active in response to an inspiratory $[8,10]$ or expiratory constraint [12] and involve cortical automatisms [10]. This leads to the concept of brainstem-cortex cooperation to sustain adequate ventilation. In patients with congenital central alveolar hypoventilation, it has been postulated that the cortical drive to breathe evidenced during wakefulness compensates for the deficient brainstem respiratory central pattern generators that contribute to the maintenance of ventilation during wakefulness [7]. In patients with severe obstructive apnoea, it has been postulated that the cortical drive to breathe contributes to overcome the increased upper airway resistance and thus explains the absence of upper airway collapse during wakefulness [11]. In patients suffering from ALS (this study), the cortical drive to breathe could compensate for diaphragm weakness through the recruitment of inspiratory muscles other than the diaphragm, and that would be cortically driven (at least in part). This is supported by the fact that those of our patients who exhibited pre-inspiratory potentials had higher inspiratory muscle activity than those who did not (table 2). More importantly, it is also supported by the fact that NIV abolished or markedly attenuated pre-inspiratory potentials when present (figs 1-3). This establishes causality regarding the respiratory origin of the observed cortical activity. Of note, while all patients exhibited inspiratory neck muscle recruitment, only eight out of 14 patients had pre-inspiratory potentials. This could indicate that our EEG approach was insufficiently sensitive to detect pre-inspiratory potentials in some cases or that other cortical mechanisms were at play. It could also mean that cortico-subcortical cooperation is only one of the possible pathways for compensatory respiratory neuroplasticity in ALS. Other possible pathways could involve brainstem plasticity, allowing increased output of preserved medullary premotor neurons to override the impact of decreased respiratory motor neuron numbers, or "redistribution" of the descending neural drive to extradiaphragmatic inspiratory muscles (i.e. neck muscles and intercostals) to compensate for the loss of phrenic motor neurons $[44,45]$.

\section{Heterogeneity among patients}

Pre-inspiratory potentials were present in eight of our patients and were absent in six. While this did not translate into a survival difference, those patients who did not exhibit pre-inspiratory potentials had more severe motor impairment, a longer disease duration and more severe respiratory muscle weakness (table 1). In these patients, the lack of respiratory-related cortical activity could be due to disease progression, resulting in the loss of previously established neuroplasticity. Abnormal recruitment of nonprimary motor regions, including the SMA, has been described in ALS patients during manual tasks [21-24, 46], but these changes are not observed in patients with more advanced forms of the disease. This has been attributed to motor neuron loss, which could extend beyond the primary motor cortex and involve the SMA. Similarly, electrophysiological substrates of movement preparation are altered in patients with 
TABLE 2 Inspiratory neck muscle electromyographic data

\begin{tabular}{|c|c|c|c|}
\hline $\begin{array}{l}\text { Overall } \\
\text { population }\end{array}$ & $\mathrm{PIP}^{+}$ & $\mathrm{PIP}^{-}$ & $\begin{array}{c}\text { p-value } \\
\left(\mathrm{PIP}^{+} \text {versus } \mathrm{PIP}^{-}\right)\end{array}$ \\
\hline
\end{tabular}

\begin{tabular}{|c|c|c|c|c|}
\hline Subjects n & 14 & 8 & 6 & \\
\hline \multicolumn{5}{|l|}{ EMGmax mV } \\
\hline Spontaneous breathing & $12.3(6.1-18.9)$ & $13.8(8.6-28.1)$ & $5.7(3.1-11.9)$ & 0.06 \\
\hline NIV & $1.2(0.6-5.6)$ & $1.6(0.7-5.8)$ & $0.6(0.4-3.4)$ & 0.11 \\
\hline $\begin{array}{l}\text { p-value (spontaneous } \\
\text { breathing versus NIV) }\end{array}$ & $<0.001$ & 0.02 & 0.06 & \\
\hline \multicolumn{5}{|l|}{ EMGAUC $\times f R \mathrm{mV}^{2} \cdot \mathrm{min}^{-1}$} \\
\hline Spontaneous breathing $\times 10^{5}$ & $3.17(1.0-4.4)$ & $3.41(2.2-7.1)$ & $1.03(0.6-2.8)$ & $<0.001$ \\
\hline NIV $\times 10^{4}$ & $2.1(6.1-7.0)$ & $2.5(1.3-8.9)$ & $6.4(5.9-2.4)$ & 0.20 \\
\hline $\begin{array}{l}\text { p-value (spontaneous } \\
\text { breathing versus NIV) }\end{array}$ & $<0.001$ & 0.02 & 0.06 & \\
\hline
\end{tabular}

Data are presented as $\mathrm{n}$ or median (interquartile range), unless otherwise stated. PIP: preinspiratory potentials; EMGmax: peak value of the averaged inspiratory neck muscle electromyographic activity; NIV: noninvasive ventilation; EMGAUc: area under the curve of the averaged inspiratory neck muscle electromyographic activity; $f R$ : respiratory frequency.

advanced ALS [25], and premotor potentials are more severely attenuated in ALS patients with intense spasticity [26]. In addition, our patients without pre-inspiratory potentials used NIV for longer daily durations (table 1). Mechanical ventilation has been shown to acutely depress diaphragm motor cortex excitability in healthy subjects [43] and in patients with chronic obstructive pulmonary disease [27]. It can be postulated that NIV itself could have had a long-term inhibitory effect on respiratory-related cortical activity in our patients. Finally, the presence or absence of pre-inspiratory potentials could reflect an EEG biomarker of ALS phenotypic heterogeneity [28].

\section{Influence on dyspnoea}

Figure 5 depicts a correlation between NIV-induced relief in dyspnoea and NIV-induced decrease in inspiratory neck muscle EMG activity. Such a relationship is expected based on experimental [29] and clinical [30] data. Here, it is mostly driven by data from patients exhibiting pre-inspiratory potentials. These patients experienced more severe dyspnoea than those without pre-inspiratory potentials (table 3), although their respiratory muscle strength was less severely altered and their neurological impairment less marked (table 1). This supports the idea that respiratory-related cortical activity may be among the determinants of dyspnoea, a concept that has already been proposed on the basis of experimental studies [8, 12]. The present data indicate that such a relationship can also exist in a clinical setting of dyspnoea. In this view,

FIGURE 4 Assessment of dyspnoea using the Multidimensional Dyspnea Profile (MDP) during spontaneous breathing and noninvasive ventilation For details, see the methods section and [14]. A1: immediate affective component of the score; SQ: sensory quality. * $p<0.05$.

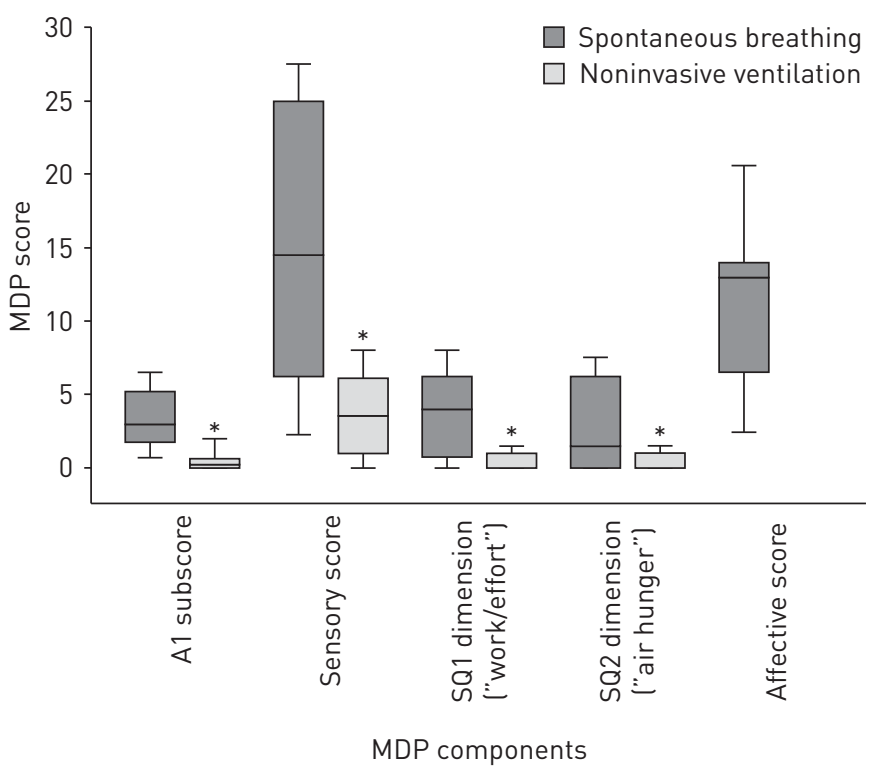


TABLE 3 Results of Multidimensional Dyspnea Profile during spontaneous breathing and noninvasive ventilation (NIV) according to the pre-inspiratory cortical activity

Overall population

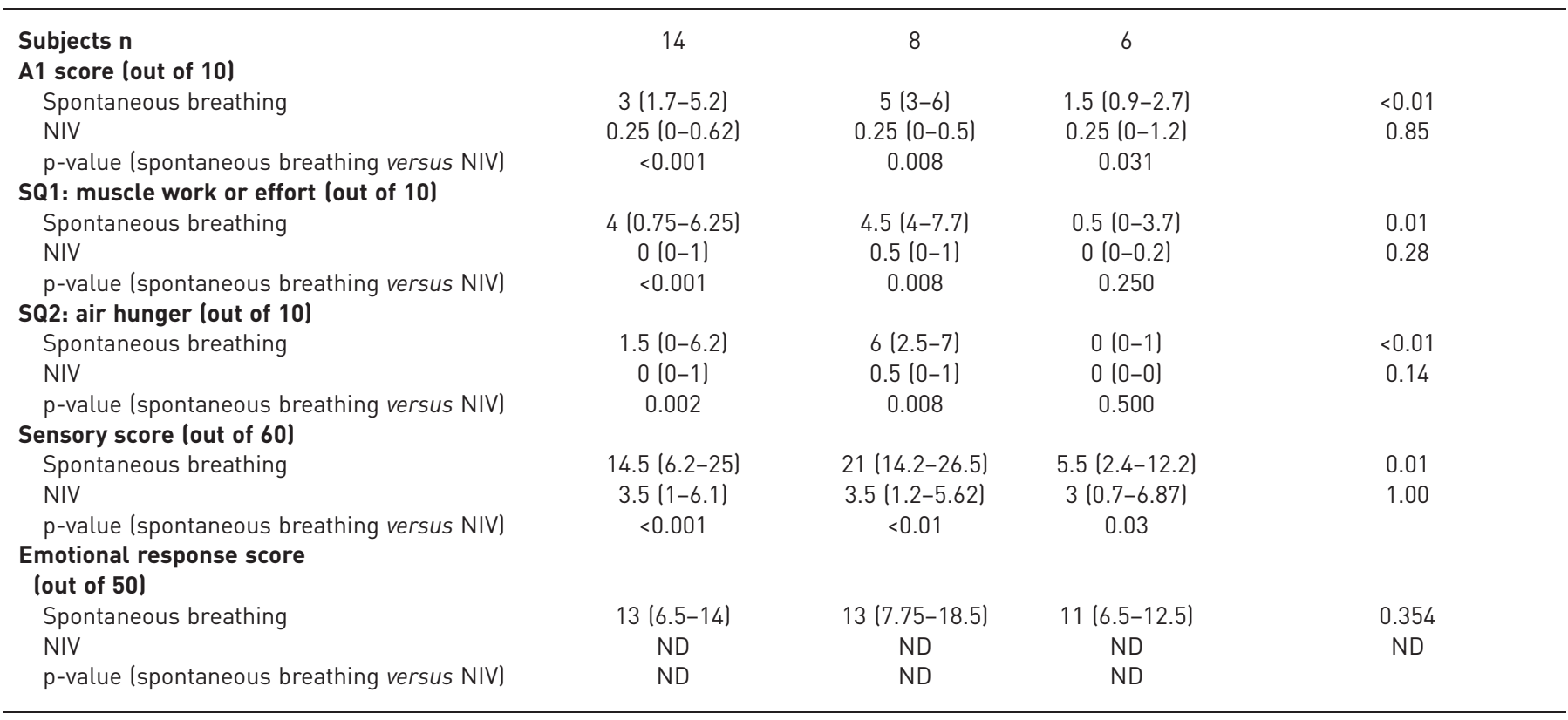

Data are presented as $\mathrm{n}$ or median (interquartile range), unless otherwise stated. PIP: pre-inspiratory potentials; SQ: sensory quality; ND: not determined.

dyspnoea would appear as one of the negative consequences of the cortical compensation of diaphragm weakness. Those of our patients without pre-inspiratory potentials were less dyspnoeic, which would mean that the disappearance of dyspnoea with time would be of poor prognosis in ALS.

One possible interpretation of the relationship between dyspnoea and respiratory-related cortical activity relies on the "corollary discharge" theory of dyspnoea [31]. This theory involves an imbalance between the global motor respiratory output as "copied" to the cerebral cortex and respiratory afferents. From this perspective, it is interesting to note that the SMA, which is most probably involved in the genesis of pre-inspiratory potentials $[10,32]$, receives respiratory afferents $[33,34]$ and is involved in the prediction of the sensory consequences of movement [35]. Another possible interpretation involves connections between the SMA and the limbic cortex [36], which also receives respiratory afferents [37] and is markedly implicated in the pathogenesis of inspiratory

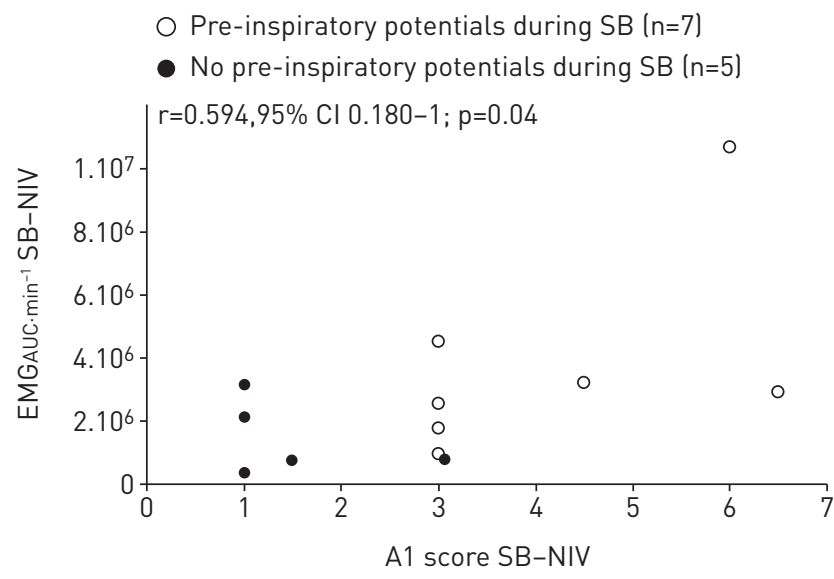

FIGURE 5 Correlation between the intensity of the noninvasive ventilation (NIV)-induced relief of dyspnoea (A1 score of the Multidimensional Dyspnea Profile) and the magnitude of the NIV-induced decrease in inspiratory neck muscle electromyogram (EMG) activity, as recorded by the neck surface electrode. SB: spontaneous breathing; SB-NIV: difference between the two conditions; EMGAUC $\mathrm{min}^{-1}$ : area under the inspiratory neck muscle EMG envelope after signal processing (see the methods section). 
loading-related dyspnoea [38]. Of note, the increased activation of cortical areas involved in movement preparation has been described in ALS during manual tasks and interpreted as resulting from compensatory neuroplasticity (see earlier) [21-24, 46]. It has been associated with increased activity in the anterior insula [46].

\section{Study limitations}

The main limitations of this study are the small size of the population and its very specific nature (i.e. ALS patients with overt respiratory failure). We acknowledge that this may reduce the generalisability of our results. Further studies of the cortical contribution to the neural drive to breathe should therefore be conducted in patients with earlier forms of ALS. Additionally, longitudinal studies are necessary to provide a relevant picture, more clearly understand how this phenomenon can explain certain features of ALS-related respiratory failure and determine whether these findings have prognostic or practical implications (e.g. to guide NIV management). Meanwhile, we believe that our results have proof-of-concept value and can be hypothesis-generating.

\section{Perspectives}

This study provides novel information about breathing control in ALS-related respiratory failure, as it demonstrates a cortical contribution to the neural drive to breathe in some patients and suggests that this contribution plays a role in the pathogenesis of dyspnoea. In view of the cortical connectivity breakdown that is associated with sleep [39], these results are highly relevant to the respiratory-related alterations in sleep quality that are typical of ALS. They could also open innovative research avenues regarding ALS-related cognitive impairment. Firstly, certain forms of ALS-related cognitive impairment have recently been related to functional abnormalities within the dorsomedial and dorsolateral prefrontal cortices and in the SMA [47] with altered amygdala-SMA connectivity [48]. Secondly, a relationship between cognitive and executive performances and respiratory-related cortical activity and connectivity was documented in a patient with congenital central hypoventilation who exhibited pre-inspiratory potentials during wakefulness [7] and who obtained better results on psychometric testing during mechanical ventilation than during spontaneous breathing [49]. The need to mobilise the SMA to maintain ventilation may not only accentuate dyspnoea but may also interfere with cognitive performance in the presence of pre-existing ALS-related SMA dysfunction, or even more simply, with motor performance [50]. These hypotheses could be tested by assessing the effects of NIV on cognitive and motor performance.

\section{References}

1 Bourke SC, Shaw PJ, Gibson GJ. Respiratory function vs sleep-disordered breathing as predictors of QOL in ALS. Neurology 2001; 57: 2040-2044.

2 Gil J, Funalot B, Verschueren A, et al. Causes of death amongst French patients with amyotrophic lateral sclerosis: a prospective study. Eur J Neurol 2008; 15: 1245-1251.

3 Gordon PH, Salachas F, Bruneteau G, et al. Improving survival in a large French ALS center cohort. J Neurol 2012; 259: 1788-1792.

4 Attali V, Mehiri S, Straus C, et al. Influence of neck muscles on mouth pressure response to cervical magnetic stimulation. Am J Respir Crit Care Med 1997; 156: 509-514.

5 Similowski T, Attali V, Bensimon G, et al. Diaphragmatic dysfunction and dyspnoea in amyotrophic lateral sclerosis. Eur Respir J 2000; 15: 332-337.

6 Arnulf I, Similowski T, Salachas F, et al. Sleep disorders and diaphragmatic function in patients with amyotrophic lateral sclerosis. Am J Respir Crit Care Med 2000; 161: 849-856.

7 Tremoureux L, Raux M, Hudson AL, et al. Does the supplementary motor area keep patients with Ondine's curse syndrome breathing while awake? PLoS One 2014; 9: e84534.

8 Raux M, Ray P, Prella M, et al. Cerebral cortex activation during experimentally induced ventilator fighting in normal humans receiving noninvasive mechanical ventilation. Anesthesiology 2007; 107: 746-755.

9 Raux M, Straus C, Redolfi S, et al. Electroencephalographic evidence for pre-motor cortex activation during inspiratory loading in humans. J Physiol 2007; 578: 569-578.

10 Raux M, Tyvaert L, Ferreira M, et al. Functional magnetic resonance imaging suggests automatization of the cortical response to inspiratory threshold loading in humans. Respir Physiol Neurobiol 2013; 189: 571-580.

11 Launois C, Attali V, Georges M, et al. Cortical drive to breathe during wakefulness in patients with obstructive sleep apnea syndrome. Sleep 2015; 38: 1743-1749.

12 Morawiec E, Raux M, Kindler F, et al. Expiratory load compensation is associated with electroencephalographic premotor potentials in humans. J Appl Physiol 2015; 118: 1023-1030.

13 Brooks BR, Miller RG, Swash M, et al. El Escorial revisited: revised criteria for the diagnosis of amyotrophic lateral sclerosis. Amyotroph Lateral Scler Other Motor Neuron Disord 2000; 1: 293-299.

14 Banzett RB, O’Donnell CR, Guilfoyle TE, et al. Multidimensional dyspnea profile: an instrument for clinical and laboratory research. Eur Respir J 2015; 45: 1681-1691.

15 Meek PM, Banzett R, Parshall MB, et al. Reliability and validity of the multidimensional dyspnea profile. Chest 2012; 141: 1546-1553.

16 Raux M, Tremoureux L, Couturier A, et al. Simplified recording technique for the identification of inspiratory premotor potentials in humans. Respir Physiol Neurobiol 2010; 171: 67-70.

17 Hug F, Raux M, Morelot-Panzini C, et al. Surface EMG to assess and quantify upper airway dilators activity during non-invasive ventilation. Respir Physiol Neurobiol 2011; 178: 341-345.

18 Hug F, Raux M, Prella M, et al. Optimized analysis of surface electromyograms of the scalenes during quiet breathing in humans. Respir Physiol Neurobiol 2006; 150: 75-81. 
19 Feldman JL, Del Negro CA, Gray PA. Understanding the rhythm of breathing: so near, yet so far. Annu Rev Physiol 2013; 75: 423-452.

20 McKay LC, Adams L, Frackowiak RS, et al. A bilateral cortico-bulbar network associated with breath holding in humans, determined by functional magnetic resonance imaging. Neuroimage 2008; 40: 1824-1832.

21 Konrad C, Henningsen H, Bremer J, et al. Pattern of cortical reorganization in amyotrophic lateral sclerosis: a functional magnetic resonance imaging study. Exp Brain Res 2002; 143: 51-56.

22 Konrad C, Jansen A, Henningsen H, et al. Subcortical reorganization in amyotrophic lateral sclerosis. Exp Brain Res 2006; 172: 361-369.

23 Han J, Ma L. Functional magnetic resonance imaging study of the brain in patients with amyotrophic lateral sclerosis. Chin Med Sci J 2006; 21: 228-233.

24 Cosottini M, Pesaresi I, Piazza S, et al. Structural and functional evaluation of cortical motor areas in amyotrophic lateral sclerosis. Exp Neurol 2012; 234: 169-180.

25 Thorns J, Wieringa BM, Mohammadi B, et al. Movement initiation and inhibition are impaired in amyotrophic lateral sclerosis. Exp Neurol 2010; 224: 389-394.

26 Westphal KP, Heinemann HA, Grözinger B, et al. Bereitschaftspotential in amyotrophic lateral sclerosis (ALS): lower amplitudes in patients with hyperreflexia (spasticity). Acta Neurol Scand 1998; 98: 15-21.

27 Hopkinson NS, Sharshar T, Dayer MJ, et al. The effect of acute non-invasive ventilation on corticospinal pathways to the respiratory muscles in chronic obstructive pulmonary disease. Respir Physiol Neurobiol 2012; 183: 41-47.

28 Swinnen B, Robberecht W. The phenotypic variability of amyotrophic lateral sclerosis. Nat Rev Neurol 2014; 10: 661-670.

29 Chiti L, Biondi G, Morelot-Panzini C, et al. Scalene muscle activity during progressive inspiratory loading under pressure support ventilation in normal humans. Respir Physiol Neurobiol 2008; 164: 441-448.

30 Schmidt M, Kindler F, Gottfried SB, et al. Dyspnea and surface inspiratory electromyograms in mechanically ventilated patients. Intensive Care Med 2013; 39: 1368-1376.

31 Lansing RW, Gracely RH, Banzett RB. The multiple dimensions of dyspnea: review and hypotheses. Respir Physiol Neurobiol 2009; 167: 53-60.

32 Ball T, Schreiber A, Feige B, et al. The role of higher-order motor areas in voluntary movement as revealed by high-resolution EEG and fMRI. Neuroimage 1999; 10: 682-694.

33 Logie ST, Colrain IM, Webster KE. Source dipole analysis of the early components of the RREP. Brain Topogr 1998; 11: 153-164.

34 von Leupoldt A, Keil A, Chan PY, et al. Cortical sources of the respiratory-related evoked potential. Respir Physiol Neurobiol 2010; 170: 198-201.

35 Makoshi Z, Kroliczak G, van Donkelaar P. Human supplementary motor area contribution to predictive motor planning. J Mot Behav 2011; 43: 303-309.

36 Hoffstaedter F, Grefkes C, Caspers S, et al. The role of anterior midcingulate cortex in cognitive motor control: evidence from functional connectivity analyses. Hum Brain Mapp 2014; 35: 2741-2753.

37 Straus C, Zelter M, Derenne JP, et al. Putative projection of phrenic afferents to the limbic cortex in humans studied with cerebral-evoked potentials. J Appl Physiol 1997; 82: 480-490.

38 Peiffer C, Poline JB, Thivard L, et al. Neural substrates for the perception of acutely induced dyspnea. Am J Respir Crit Care Med 2001; 163: 951-957.

39 Massimini M, Ferrarelli F, Huber R, et al. Breakdown of cortical effective connectivity during sleep. Science 2005; 309: 2228-2232.

40 Evans KC, Shea SA, Saykin AJ. Functional MRI localisation of central nervous system regions associated with volitional inspiration in humans. J Physiol 1999; 520: 383-392.

41 Smejkal V, Druga R, Tintera J. Brain activation during volitional control of breathing. Physiol Res 2000; 49: 659-663.

42 Tremoureux L, Raux M, Ranohavimparany A, et al. Electroencephalographic evidence for a respiratory-related cortical activity specific of the preparation of prephonatory breaths. Respir Physiol Neurobiol 2014; 204: 64-70.

43 Sharshar T, Ross ET, Hopkinson NS, et al. Depression of diaphragm motor cortex excitability during mechanical ventilation. J Appl Physiol 2004; 97: 3-10.

44 Johnson RA, Mitchell GS. Common mechanisms of compensatory respiratory plasticity in spinal neurological disorders. Respir Physiol Neurobiol 2013; 189: 419-428.

45 Nichols NL, Van Dyke J, Nashold L, et al. Ventilatory control in ALS. Respir Physiol Neurobiol 2013; 189: 429-437.

46 Kew JJ, Leigh PN, Playford ED, et al. Cortical function in amyotrophic lateral sclerosis. A positron emission tomography study. Brain 1993; 116: 655-680.

47 Carluer L, Mondou A, Buhour MS, et al. Neural substrate of cognitive theory of mind impairment in amyotrophic lateral sclerosis. Cortex 2015; 65: 19-30.

48 Passamonti L, Fera F, Tessitore A, et al. Dysfunctions within limbic-motor networks in amyotrophic lateral sclerosis. Neurobiol Aging 2013; 34: 2499-2509.

49 Sharman M, Gallea C, Lehongre K, et al. The cerebral cost of breathing: an FMRI case-study in congenital central hypoventilation syndrome. PLoS One 2014; 9: e107850.

$50 \mathrm{Li} \mathrm{H}$, Chen $\mathrm{Y}$, Li Y, et al. Altered cortical activation during action observation in amyotrophic lateral sclerosis patients: a parametric functional MRI study. Eur Radiol 2015; 25: 2584-2592. 